\title{
Una propuesta de rendición de cuentas en las organizaciones comunitarias en el estado Mérida (Venezuela)*
}

\section{A proposal for accountability in community organizations in Mérida (Venezuela)}

\author{
Wolff Berenice** \\ Díaz Katty***
}

Recibido: 2 de septiembre de 2012

Aprobado: 16 de enero de 2013

\section{Resumen}

La participación comunitaria se encuentra en la Constitución de la República Bolivariana de Venezuela como un derecho. Los participantes de los consejos comunales (en adelante, $\mathrm{CG}$ ) despliegan actos de autoridad que deben ser sometidos a las reglas de responsabilidad que se encuentran en las leyes del Estado venezolano. Esta investigación

* Este artículo es extraído de los resultados del trabajo de grado presentado como requisito parcial para optar por el título de Maestría en Ciencias Contables, adscrito al proyecto financiado por el Consejo de Desarrollo Humanístico (CDCHTA) de la Universidad de Los Andes con el código E-304-09-09-EM. Cómo citar este artículo: Wolf, B., \& katty,D. (2013). Una propuesta de rendición de cuentas en las organizaciones comunitarias en el estado Mérida (Venezuela). Revista CIFE, 15 (22), pp. 161-187.

** Licenciada en Contaduría Pública. Magister en Ciencias Contables. Universidad de Los Andes (ULA). Av. Tulio Febres Cordero Edificio Administrativo de la ULA. Departamento de Nómina. +58 02742402752 bwolf@ula.ve.

*** Economista. Magister en Economía. Universidad de Los Andes. Escuela de Economía. Av. Las Américas. Complejo Liria. Edificio H Tercer piso Departamento de Economía. +582742401017 kattymar@ula.ve. 
ISSN: 0124-3551 / Año 15, No 22 / enero-junio / pp. 161-187

estudiará el proceso de rendición de cuentas de los recursos financieros otorgados a los CG del municipio Rangel del estado Mérida. El resultado es que los CG surgen como una tipología de organización comunitaria que obligó a sus miembros a adaptarse a una estructura de gestión pública novedosa y sin acompañamiento público. Para ello, se proponen un conjunto de estrategias que contribuirán a formalizar este proceso y mejorar su desempeño.

Palabras clave: Consejos comunales; rendición de cuentas; participación comunitaria; organizaciones comunitarias.

Clasificación JEL: JEL: L0, L3, H7

\section{Abstract}

Community participation is in the Constitution of the Bolivarian Republic of Venezuela as a right. Participants in the CG display acts of authority that should be subject to the rules of liability found in the laws of the State of Venezuela. This research will examine the process Accountability of financial resources granted to the municipality CC Rangel Merida state. The result is that the CG emerge as a type of community organization that forced its members to adapt to a new governance structure and without public support. To this end, we propose a set of strategies that will help formalize this process and improve its performance.

Keywords: Community councils; accountability; community participation; community organizations.

Classification JEL: JEL: L0, L3, H7 


\section{Introducción}

La evolución de la democracia en el Estado venezolano, permitió que este incursionara en el campo de la planificación pública y los procesos contables y de control. Venezuela en 1958, emprende una reforma presupuestaria y contable con la creación del "Sistema de Planificación" (Decreto 492 del 30 de diciembre de 1958) y la Comisión de la Administración Pública Nacional (Decreto 287, del 27 de junio de 1958). Esta reforma incluyó el presupuesto por programas como un anexo explicativo de la Ley Orgánica del Régimen Presupuestario y, para darle cumplimiento a esta ley, se creó la oficina central de presupuesto (OCEPRE), órgano rector de este sistema, con una estructura administrativa similar a la que poseía este organismo.

El área de presupuestos regionales implementó la figura de coordinadores regionales con sede en el interior del país, ellos tenían como funciones: asistir a las corporaciones regionales, gobernaciones de estado y los concejos municipales, así, en 1981, su estructura fue adecuada a los esquemas previstos en el plan de la Nación para ese momento. Posteriormente, al sancionarse la Ley Orgánica de la Administración Financiera del Sector Público (LOAFSP), se fueron integrando los sistemas de presupuesto, crédito público, tesorería y contabilidad de la administración financiera del Estado. Igualmente, se anexaron los sistemas tributario y de administración de bienes regulados por otras leyes, los cuales pasaron a formar parte integral de la oficina nacional de presupuesto (ONAPRE).

En la década de 1980, se inició un proceso de descentralización de la administración pública nacional, el cual pretendía que las gobernaciones de los estados y los municipios emprendieran el diagnóstico de sus potencialidades. A partir de allí, se generaron propuestas de proyectos, el manejo de recursos económicos y la toma de decisiones en materia de política económica siguiendo los lineamientos nacionales. A partir de 1988, esta iniciativa comienzó a ejecutarse, permitiendo el fortaleciendo de la autonomía y las competencias locales, tanto de las gobernaciones como de las alcaldías. En este sentido, se iniciaron las elecciones de alcaldes y gobernadores, surgiendo a partir de esta experiencia un nuevo liderazgo que le otorgó poder a las regiones y municipios.

De acuerdo con Sánchez (2002), en este proceso de descentralización ocurrieron cambios en el área de las finanzas públicas:

(...) (a) Se aumentó el Situado Constitucional del 15\% al $20 \%$ de los ingresos ordinarios; (b) El situado municipal: el $20 \%$ de los ingresos ordinarios de los Estados, es una cifra sustancialmente mayor que la que existía en el esquema existente antes de aprobarse la ley de descentralización; (c) Se abrió la puerta a nuevos impuestos y tasas en la Ley de Descentralización (1989); (d) En 1993 se creó el Fondo Intergubernamental para la 
Descentralización (FIDES); (e) En 1996 se aprobó la Ley de Asignaciones Económicas Especiales (LAEE). (Sánchez, 2002, p. 24)

Moreno (2007), señala que a partir de la descentralización se incentivó la participación política en las elecciones, se profundizó en valores democráticos, se elevó la eficiencia de las políticas públicas, y se avanzó en un proceso de participación de la ciudadanía en el país, luchando contra el centralismo. Así mismo, este proceso de descentralización fue absorbido por el sistema político, lo que reprodujo un antiguo esquema de absorción de renta que trasladó a los gobiernos locales prácticas que ponían en riesgo el proceso.

Ante este escenario, Moreno (2007), vislumbró el fracaso del cumplimiento de las políticas de descentralización, por lo que el Gobierno Nacional, desde la institucionalidad, adoptó medidas para corregir este proceso e introdujo como opción la participación ciudadana individual o colectiva. En tal sentido, esta propuesta involucró nuevos actores en las decisiones y en los actos que se producían desde los organismos públicos, con el sentido de colaboración, cooperación, seguimiento, control y evaluación de los ciudadanos y las organizaciones comunitarias de la sociedad, que reciben los bienes y servicios que el Estado está obligado a generar para la satisfacción de sus necesidades.

De acuerdo con lo anteriormente expuesto, se tornó novedoso la incorporación de la participación ciudadana en el marco legal vigente a partir de la Constitución de la República Bolivariana de Venezuela (1999), en la que se fortaleció y se concibió la misma, como un deber y un derecho de todo ciudadano y ciudadana a ejercer acciones de control fiscal de la gestión pública, y se fundamentó en el fortalecimiento de la democracia participativa, protagónica y corresponsable. A este nivel, los líderes que ejercen el poder público y los ciudadanos son responsables de la calidad de vida que en su entorno obtengan, y desde esta perspectiva, el municipio es la unidad político territorial en donde los administradores y administrados convergen con mayor cercanía.

El ordenamiento jurídico venezolano, promulgado con la Constitución de la República Bolivariana de Venezuela (1999), se ha adecuado a los postulados de la participación ciudadana, con la incorporación de la normativa que rige en cada caso. En este sentido los municipios son las unidades primarias de gestión pública nacional que vienen a ser el lugar apropiado para que los ciudadanos realicen su deber y puedan ejercer el derecho de participar en el seguimiento, control y evaluación de gestión de los líderes que ejercen el poder público municipal. Todo ello, motivado por la consecución de una mejora constante y sostenible en la calidad de vida. No obstante, la participación ciudadana en la gestión pública, representada en la ciudadanía, tiene un conjunto de derechos y deberes con los que puede participar. Por lo tanto, esta representa un factor prioritario en el control de la gestión pública, debido a que, mediante este mecanismo la sociedad contribuye con el seguimiento, vigilancia, control y evaluación del comportamiento de 
quienes dirigen las entidades públicas en el cumplimiento de las metas y objetivos de su competencia.

En este contexto, según la Constitución de la República Bolivariana de Venezuela (1999), artículo 168, establece que los municipios se convierten en espacios originarios para la participación ciudadana, por constituir “...la unidad política primaria de la organización nacional...". Así mismo, la Ley Orgánica del Poder Público Municipal (LOPPM), en su artículo $2^{\circ}$, ratifica la obligatoriedad que tienen los municipios de incorporar en sus actuaciones la participación ciudadana de manera efectiva, suficiente y oportuna, en la definición y ejecución de la gestión pública, en el control y evaluación de sus resultados. Y, la LOPPM, artículo $7^{\circ}$, señala que: "el municipio y las demás entidades locales, conforman espacios primarios para la participación ciudadana en la planificación, diseño, ejecución, control y evaluación de la gestión pública, los órganos del Municipio y demás entes locales, deberán crear los mecanismos, para garantizar la participación de las comunidades y grupos sociales organizados en su jurisdicción."

Por otra parte, sobre el control fiscal, la Ley Orgánica de la Contraloría General de la República y del Sistema Nacional de Control Fiscal (2001), en el artículo $6^{\circ}$ establece: "Los órganos que integran el Sistema Nacional de Control Fiscal adoptarán las medidas necesarias para fomentar la participación ciudadana en el ejercicio del control sobre la gestión pública", igualmente, el artículo 24 de la misma ley, en su punto 4 señala que el Sistema Nacional de Control Fiscal lo integran: "(...) 4. Los ciudadanos, en el ejercicio de su derecho a la participación en la función de control de la gestión pública".

De acuerdo con el artículo 23 de la Ley Orgánica de la Contraloría General de la República y del Sistema de Control Fiscal, la administración pública tiene por objeto: "...fortalecer la capacidad del Estado para ejecutar eficazmente su función de gobierno, lograr la transparencia en el manejo de los recursos del sector público y establecer la responsabilidad por la comisión de irregularidades relacionadas con la gestión de las entidades..." sujetas al sistema de control fiscal nacional.

En el caso de los municipios, el sistema de control fiscal lo constituyen: la contraloría municipal, la máxima autoridad del municipio ejercida por el alcalde o alcaldesa; el concejo municipal, la unidad de auditoría interna, niveles directivos, gerenciales y operativos de la administración, y los ciudadanos y ciudadanas individual o colectivamente, a través de los consejos locales de planificación pública, consejos parroquiales, consejos comunales $(\mathrm{CG})$, contralorías sociales y comunidad organizada.

En relación con la participación de los ciudadanos en el control fiscal de la gestión pública municipal, el artículo 253 de la Ley Orgánica del Poder Público Municipal (2005), establece:

"La participación protagónica del pueblo en la formación, ejecución y control de la gestión pública municipal es el medio necesario para garantizar su completo desarrollo 
tanto individual como colectivo, dentro del municipio. Las autoridades municipales deberán promover y garantizar la participación de los ciudadanos y ciudadanas en la gestión pública y facilitar los medios y procedimientos para que los derechos de participación se materialicen de manera efectiva, suficiente y oportuna".

De esta manera, todos los entes gubernamentales estructurados desde el gobierno nacional, gobernaciones y alcaldías, formados como organizaciones públicas tradicionales encuentran dificultades para atender este proceso y dar respuestas para impulsar el desarrollo local por esta vía. Así, Delgado (1995), explica que existe una debilidad en la capacidad institucional del gobierno, manifestada de diversas maneras y con dimensiones e impactos distintos, tales como:

“(...) (a) Demandas ciudadanas exigiendo un mejor y más transparente desempeño gubernamental; (b) Mayores expectativas sociales sobre las acciones de gobierno, sin disposición a aumentar los costos oficiales; (c) Nuevas y fortalecidas demandas políticas, tales como democracia participativa, derechos humanos descentralización y autonomía Local; (d) Cambios profundos en la naturaleza de los problemas sociales debido a la interdependencia de sus causas y la complejidad de sus consecuencias". (p. 7)

Con la promulgación de la Ley de los Consejos Comunales (LCC), se hace factible la participación ciudadana en todas y cada una de las decisiones de la comunidad. De esta manera, y con esta ley la autoridad nacional tiene como objetivo hacer viable la participación de las comunidades, como se expresa en el artículo $2^{\circ}$ de la misma:

"Los consejos comunales en el marco constitucional de la democracia participativa y protagónica, son instancias de participación, articulación e integración entre las diversas organizaciones comunitarias, grupos sociales y los ciudadanos y ciudadanas, que permiten al pueblo organizado ejercer directamente la gestión de las políticas públicas y proyectos en la construcción de una sociedad de equidad y justicia social".

Los CG tienen acción en un conjunto de áreas como: infraestructura (vialidad, drenajes), servicios públicos (seguridad, transporte, aguas servidas, aguas blancas, alumbrado público), servicios sociales (educación, salud, recreación, alimentación, atención a la población en situación de riesgos como los niños, indigentes y ancianos), entre otros.

Las funciones principales de los CG son: la participación activa de la ciudadanía en el proceso de decisión público, la planificación comunitaria local, la gestión y administración de los recursos a nivel de las obras, las cuales se encuentran en el mínimo nivel de la categoría programática presupuestaria, que es indivisible a los fines de asignación formal de recursos financieros y que demanda su ejecución. En tal sentido, Paredes, (2006, p. 61), las caracteriza como: "obra: (a) Constituyen la unidad perfectamente individual, (b) Satisface parcialmente el propósito del proyecto, como condición necesaria, pero no suficiente para su cumplimiento, (c) Puede ser objeto de contratación separada del resto de los componentes". 
Por todo lo anteriormente expuesto, los integrantes de los $\mathrm{CG}$ se encuentran en un contexto legal que les genera responsabilidades y deberes explícitos. Por ello, es importante determinar: ¿están en capacidad operativa los CG para realizar una efectiva rendición de cuentas de los recursos utilizados? Y, ¿están las instituciones en capacidad de establecer mecanismos que permitan mejorar el seguimiento de los recursos otorgados y la ejecución de los mismos? En este sentido, para responder a estos interrogantes este trabajo estudió la experiencia particular del municipio Rangel del estado Mérida, con el objetivo de evaluar el proceso de rendición de cuentas de los recursos asignados a los CG de este municipio. Así mismo, diagnosticar cómo funcionan los mismos en conjunto con las organizaciones responsables de hacer seguimiento y dar cumplimiento del marco legal que los regula. El periodo estudiado, 2006-2009, fue determinado por el año de formación de los $\mathrm{CG}$ y la culminación del primer grupo de proyectos ejecutados.

\section{Aspectos teóricos y metodológicos}

\subsection{Aspectos generales del proceso de rendición de cuentas}

El presupuesto es uno de los componentes del plan operativo anual del sector público, a través del cual, se procura la definición concreta y la materialización de los objetivos de dicho sector, basándose en la definición de programas creados en función de la misión del organismo público que elabora el presupuesto. En muchas oportunidades la gestión de gobierno tiene la necesidad de emprender proyectos cuyos objetivos traspasan la misión del órgano público que elabora el presupuesto. Por esta razón, a partir de 2006 este por decisión del Ejecutivo Nacional, sustituye la técnica de cálculo por programas para adoptar la del presupuesto por proyectos. Así mismo, en ese mismo año se incorporó a la legislación venezolana la LCG, que menciona en el artículo $2^{\circ}$ : “...el pueblo organizado puede ejercer la gestión de politicas públicas y proyectos orientados a responder a las necesidades y aspiraciones de las comunidades...".

La concepción moderna de presupuesto está sustentada en el carácter de integridad de la técnica financiera. El presupuesto no sólo es concebido como una expresión financiera del plan de gobierno, sino como una expresión más amplia, ya que se constituye en un instrumento del sistema de planificación que refleja una política presupuestaria única.

El presupuesto público está compuesto por cuatro fases: a) formulación; b) ejecución; c) control, y d) evaluación. En la fase de control y evaluación es donde se encuentra determinada la rendición de cuentas y el control de los recursos presupuestarios asignados. Según Acevedo (2007, pp. 23-24), "la rendición de cuentas se puede entender como la acción de evaluar, juzgar, verificar o evidenciar colectivamente un esfuerzo realizado mediante el uso de los recursos para generar servicios que modifican condiciones a favor de una entidady/o personas." Por ello, la rendición de cuentas es considerada como una de las responsabilidades fundamentales de 
todos los funcionarios del sector público, mencionadas en el artículo 11 de la ley orgánica de la administración pública: "Las autoridades, funcionarios y funcionarias de la Administración Pública deberán rendir cuentas de los cargos que desempeñen, en los términos y condiciones que determine la Ley”. De esta manera, todos los organismos públicos están obligados a rendir cuentas de los recursos públicos que les son entregados.

El sistema de la administración pública venezolano es un macro sistema que se encuentra dividido en dos subsistemas denominados: administrativos y de apoyo. A su vez, estos se encuentran fraccionados en varios subsistemas, y cada uno de ellos está compuesto por sus propios elementos (Esquema 1).

Esquema 1: Sistemas de la administración pública

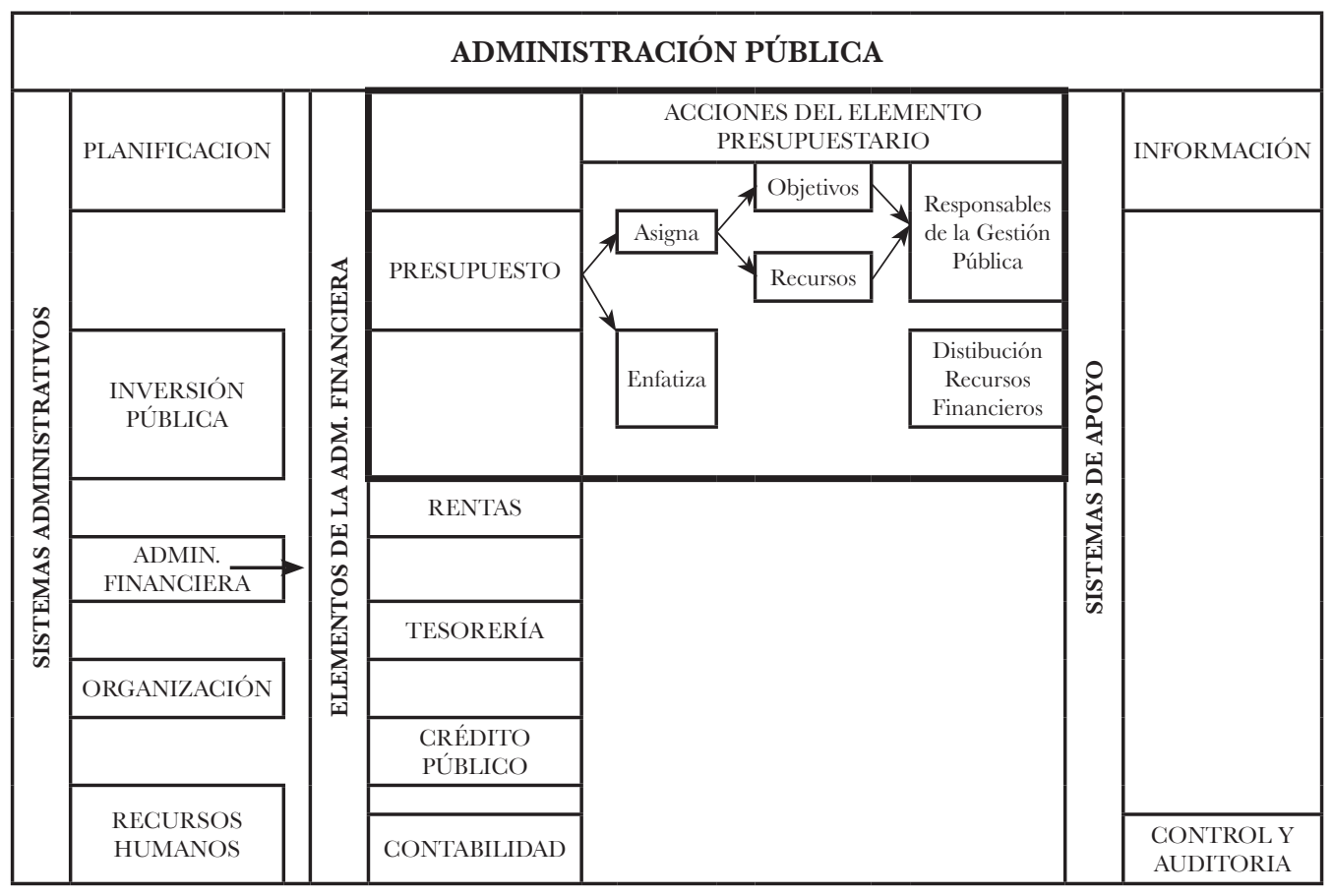

Fuente: elaborado a partir de Paredes (2006).

Este trabajo se centró en el estudio del subsistema de la administración financiera, definida por Paredes (2006, p. 34) como: “... sistema integrado que mediante la planificación, determinación, obtención, utilización, registro e información y control, persigue la eficiente gestión de los recursos financieros del Estado para la satisfacción de necesidades colectivas." y los subsistemas que lo componen (Esquema 1). En relación con estos elementos se crea el Programa de Modernización de la Administración Financiera del Estado (PROMAFE), el cual diseña y desarrolla un sistema de administración financiera integrado y confiable que optimiza los resultados de la gestión pública. En tal sentido, este sistema, dotado de un marco 
legal de aplicación, que se describe en la Ley Orgánica de la Administración Financiera del Sector Público (LOAFSP), genera información oportuna a los diferentes usuarios y es llamado: Sistema Integrado de Gestión y Control de Finanzas Públicas (SIGECOF), considerado como un sistema integrador de los sub-sistemas de presupuesto, crédito público, tesorería, contabilidad, tributario y de administración de bienes. Por ello, esta estructura es la más importante dentro de la administración financiera del sector público, siendo regido por un órgano rector denominado: Oficina Nacional de Contabilidad Pública (ONCOP).

Las características del Sistema Contable del Sector Público, se encuentran definidas en la LOAFSP, en sus artículos 123, 124 y 125 y en el numeral se incorporan los CC con las funciones públicas otorgadas por la LCG.

Los $\mathrm{CC}$, son creados como una asociación comunitaria, de elección popular para ejercer potestades públicas con recursos del Estado y deben estar inscritos en la Comisión Presidencial del Poder Popular.

La participación de los ciudadanos incorporados a un CC de una localidad, se convierte en un compromiso participativo, donde la premisa más importante es el cuidado de los intereses que beneficien al colectivo, visualizando acciones u omisiones legítimas. Para ello, el Estado le confiere atribuciones a la LCG, encomendándole ciertas funciones como son: la reflexión colectiva y consensos sobre intereses públicos, la administración y gestión de los recursos para hacer viable dichos intereses, que se traducen en una mejor gestión administrativa, todos estos derechos consagrados en el marco de la legalidad.

Todas estas acciones de carácter administrativo deben poseer procedimientos previos que las regulen, ya que la LCC les da a sus miembros, facultades similares a las de la administración pública, que son de obligatorio cumplimiento y enmarcadas dentro de las leyes venezolanas. Romero (2007) señala que, la rendición de cuentas es una obligación dentro de la LCC, agregando cómo deberían llevarse.

"(...) debe llevarse nota cuidadosa de las cuentas de las instituciones del microsistema financiero donde estén depositados y los reportes mensuales de las mismas, emitidos por esas instituciones. Si se tratara de un terreno debe saberse donde está ubicado, sus linderos y medidas, el documento que ampara la titularidad, etc., respecto a los bienes muebles debe tenerse un inventario de estos y se precisa su ubicación. Del lado de los gastos administrar es llevar buen archivo de facturas y demás documentos que justifiquen cualquier salida de dinero del Banco Comunal o actuación respecto a sus bienes". (p. 104)

El organismo público, Servicio Autónomo Fondo Nacional de los Consejos Comunales (SAFONACG) es el responsable de la elaboración del presupuesto y la tramitación de los recursos técnicos, financieros y no financieros que le son otorgados a los CC. Así mismo, este cuerpo depende de la Comisión Nacional Presidencial para el Poder Popular, previa solicitud de cada CG y debe velar por el buen uso de los recursos otorgados. 
Con relación al financiamiento los CG reciben apoyo financiero de diferentes organismos y, dependiendo del mismo, estos deben cumplir con algunos requisitos. Para realizar la solicitud de financiamiento de un proyecto comunal, el CG debe estar elegido y constituido formalmente en el Banco Comunal con la figura jurídica de asociación cooperativa, como lo establece la LCG en su artículo 17.

La LCG en su artículo 22, numerales 7 y 11, exige a los CC rendir cuentas y según Herrera (2008), sólo para el financiamiento de los proyectos de las mesas técnicas de agua, es exigido este requisito, en los casos de otros entes financiadores, se exige sólo una relación de gastos.

Una manera de evaluar los programas gubernamentales es la rendición de cuentas, definida por Acevedo (2007, pp. 23-24) como: "se puede entender como la acción de evaluar, juzgar, verificar o evidenciar colectivamente un esfuerzo mediante el uso de los recursos para generar servicios que modifican condiciones a favor de una entidad o personas". Este autor induce al seguimiento de acciones, que para el tema en estudio, están relacionadas con la ejecución de proyectos y obras en beneficio de un colectivo.

La rendición de cuentas reviste una gran importancia, en el manejo de fondos públicos de manera transparente, fortalece la institucionalidad de un país y la eficiencia en el uso de los recursos. En tal sentido, la Contraloría General de la República Bolivariana de Venezuela (2004, p. 22) menciona: (...) "transparencia no solo hace referencia a la exposición de cifras, datos, referencia, cuadros; se apunta mas a la gestión, a la información precisa y clara que se requiere para la toma de decisiones y a la posterior rendición de cuentas". El mismo párrafo concluye diciendo: "...la transparencia y la rendición de cuentas son requisitos indispensable para el control ciudadano". De esta manera, este proceso es requisito indispensable para poder ejecutar el control.

Acevedo (2007, p. 27) señala que, entre los aspectos importantes de la rendición de cuentas se encuentran: a) la orientada a los resultados; b) no tiene sentido sin consecuencias; c) mejora el desempeño de la organización. Para el caso de los CG, es importante desarrollar el sentido de pertenencia en cada uno de sus miembros, lo cual implica: compromiso, involucramiento, empoderamiento, acuerdo, inversión, avance e incentivos.

De acuerdo con Acevedo (2007, pp. 43-46), los aspectos que guían los enfoques de la rendición de cuentas deben demostrar las debilidades de los sistemas actuales y proponer otros que reviertan las condiciones negativas de la misma (Cuadro 1). De esta manera, la rendición de cuentas apoya las habilidades de organizaciones privadas o públicas para: a) establecer responsables y metas medibles; b) planificar lo que se requiere hacer para lograr las metas; c) ejecutar el trabajo y monitorear el progreso; d) reportar los resultados; e) evaluar resultados; y f) proporcionar feedback (Acevedo, 2007, pp. 52-53). 
Guadro 1. Enfoques actuales y propuestos para la rendición de cuentas

\begin{tabular}{l|l|}
\multicolumn{1}{|c|}{ Enfoques actuales } & \multicolumn{1}{c}{ Enfoques propuestos } \\
\hline $\begin{array}{l}\text { Se realiza con variables económicas. } \\
\text { Ausencia de estructura uniforme. }\end{array}$ & Con indicadores económicos, cronológicos e intangibles. \\
\hline Consignación tardía de los informes & Oportunidad de la consignación de la información. \\
\hline $\begin{array}{l}\text { Ausencia de hallazgos. } \\
\text { Identificación de hallazgos como medio de superación de } \\
\text { situaciones críticas. }\end{array}$ \\
\hline $\begin{array}{l}\text { indicadores y su cálculo. } \\
\text { Ausencia de vinculación de } \\
\text { indicadores. }\end{array}$ & $\begin{array}{l}\text { Establecer un concepto universal y un procedimiento para su } \\
\text { cálculo. }\end{array}$ \\
\hline $\begin{array}{l}\text { Cuadros, gráficos, reportes, informes, } \\
\text { se gestionan sin apoyo conceptual. }\end{array}$ & $\begin{array}{l}\text { Institucionalizar el principio de análisis e interpretación } \\
\text { sistémica. }\end{array}$ \\
\hline $\begin{array}{l}\text { Debe ir más allá del análisis de } \\
\text { ingresos-gastos. }\end{array}$ & $\begin{array}{l}\text { Debe incorporar variables, económicas, físicas, cronológicas e } \\
\text { intangibles para comparaciones nacionales e internacionales. }\end{array}$ \\
\hline
\end{tabular}

Fuente: elaborado a partir de Acevedo (2007, pp. 50-51).

\subsection{Metodología}

El trabajo se realizó en dos etapas, las cuales consistieron en la revisión y estudio del marco legal sobre el funcionamiento de los CG y la rendición de cuentas. La segunda etapa consistió en el trabajo de campo, lo que permitió realizar un diagnóstico de los CG del municipio en estudio y su situación con relación a la rendición de cuentas. A partir de esta diagnosis se realizó un análisis comparativo entre la situación de los CC en el cumplimiento de la rendición de cuentas y lo descrito por la administración pública nacional.

La población objeto de estudio fueron los CG del municipio Rangel del estado Mérida. En tal sentido, de acuerdo con Hurtado (2000, p. 161), el tamaño de la muestra se seleccionó a partir de la fórmula:

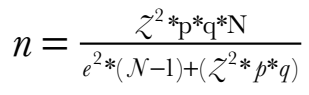

Donde:

$n$ : tamaño de la muestra.

$\mathbf{Z}^{2}$ : es el valor teórico que representa el nivel de confianza. Para el caso se escogió un nivel de confianza de 95,5\%, donde $\mathrm{Z} 2$ es igual a 4 .

e: es el error de estimación o error de muestreo que normalmente oscila entre 5 y 10 \%. En el caso del presente estudio se seleccionó un error de muestreo del $10 \%$. 
p y q: es el comportamiento del evento a medir. Como no se realizó una prueba piloto y no se tuvieron antecedentes de investigaciones anteriores, se estableció un mismo valor para p y q $(50 \%)$.

$\mathbf{N}$ : tamaño de la población, la totalidad de los CG del municipio Rangel son 90.

Cuadro 2. Datos selección de la muestra

\begin{tabular}{|l|c|c|c|}
\hline \multicolumn{4}{|c|}{ Datos muestra } \\
\hline Nivel de Confianza & Z2 & 4 \\
\hline Error de muestreo & e & $10 \%$ & \\
\hline $\begin{array}{l}\text { Comportamiento a } \\
\text { medir }\end{array}$ & p y q & $50 \%$ & $50 \%$ \\
\hline $\begin{array}{l}\text { Tamaño total de la } \\
\text { población }\end{array}$ & $\mathrm{N}$ & 90 & \\
\hline
\end{tabular}

Fuente: elaborado a partir de los datos establecidos para la determinación de la muestra.

Atendiendo a los valores descritos anteriormente, se obtuvo como resultado del tamaño de la muestra:

$$
\begin{gathered}
n=\frac{z^{2} * \mathrm{p}^{*} \mathrm{q}^{* \mathrm{~N}}}{e^{2 *(\mathcal{N}-1)+\left(Z^{2 *} p^{*} q\right)}} \\
n=\frac{4^{2} * 50 \% * 0^{*} 50 \% * 90}{10 \%{ }^{2} *(90-1)+\left(4^{*} * 50 \% * 50 \%\right)}=47,62
\end{gathered}
$$

La población en estudio correspondió a 90 CG constituidos en el municipio Rangel; el tamaño de la muestra son 47 CG. Esta cifra representa el $53 \%$ de la población en estudio:

$$
47,34 * 100 \% / 89=53 \%
$$

Así mismo, la muestra fue seleccionada siguiendo el criterio geográfico (división del municipio en parroquias). En cada una de ellas se tomó el porcentaje de la población, atendiendo al resultado del tamaño de la muestra, obteniendo los siguientes resultados: 
Una propuesta de rendición de cuentas en las organizaciones comunitarias en el estado Mérida (Venezuela)

Cuadro 3. Población del municipio Rangel y muestra por parroquia

\begin{tabular}{|l|c|c|}
\hline Parroquia & Población & Muestra \\
\hline Rangel & 31 & 16 \\
\hline Cacute & 11 & 6 \\
\hline La Toma & 14 & 7 \\
\hline Mucurubá & 16 & 8 \\
\hline San Rafael & 18 & 10 \\
\hline Total & 90 & 47 \\
\hline
\end{tabular}

Fuente: elaborado a partir de los datos establecidos para la determinación de la muestra.

Por otra parte, Tamayo (1988, p 95), clasifica una tipología denominada muestreo aleatorio simple, en el que: "El elemento más común para obtener una muestra representativa es la selección al azar-aleatoria- es decir, que cada uno de los individuos de una población tiene la misma posibilidad de ser elegido."

$\mathrm{Al}$ aplicar el muestreo definido anteriormente a la población en estudio se obtuvo como resultado los siguientes CG por parroquia.

Cuadro 4. Muestras seleccionadas del municipio Rangel por parroquia

\begin{tabular}{|c|c|c|c|}
\hline No & CONSEJO GOMUNAL & No & GONSEJO GOMUNAL \\
\hline \multicolumn{2}{|c|}{ Parroquia La Toma } & \multicolumn{2}{|c|}{ Parroquia San Rafael } \\
\hline 1 & Misasote & 1 & La bomba nueva providencia \\
\hline 2 & Carorita & 2 & Pedregal alto \\
\hline 3 & El Royal & 3 & La Mucuchache \\
\hline 4 & Valle Piñango & 4 & Las Cañaditas \\
\hline 5 & La Lagunita & 5 & La Provincia \\
\hline 6 & Frailejón andino & 6 & San Rafael del paramo \\
\hline 7 & Alto andino & 7 & Biguznos \\
\hline \multicolumn{2}{|c|}{ Parroquia Mucuruba } & 8 & Don Samuel \\
\hline 1 & Mococon Alto & 9 & El cambote \\
\hline 2 & Casco del pueblo & \multicolumn{2}{|c|}{ Parroquia Rangel (Mucuchies) } \\
\hline 3 & Mococon bajo & 1 & Corpoandes \\
\hline 4 & La ranchería & 2 & Santa Eduviges \\
\hline
\end{tabular}




\begin{tabular}{|c|l|c|l|}
\hline No & \multicolumn{1}{|c|}{ CONSEJO GOMUNAL } & No & \multicolumn{1}{|c|}{ CONSEJO COMUNAL } \\
\hline $\mathbf{5}$ & Escaguey & $\mathbf{3}$ & Chachopito \\
\hline $\mathbf{6}$ & La cruz & $\mathbf{4}$ & La Capilla de Misintá \\
\hline $\mathbf{7}$ & Mucupiche (El otro lao) & $\mathbf{5}$ & San Francisco \\
\hline $\mathbf{8}$ & Alberto Carnevali & $\mathbf{6}$ & la Hoyada \\
\hline Parroquia Cacute & $\mathbf{7}$ & Los Aposentos \\
\hline $\mathbf{1}$ & Cacutico & $\mathbf{8}$ & La Musuy \\
\hline $\mathbf{2}$ & Casco de Cacute & $\mathbf{9}$ & Misintá \\
\hline $\mathbf{3}$ & Nueva escaguey & $\mathbf{1 0}$ & Plaza Inmaculada \\
\hline $\mathbf{4}$ & Los Granates & $\mathbf{1 1}$ & San Benito de Palermo \\
\hline $\mathbf{5}$ & Los Ranchos & $\mathbf{1 2}$ & Av Independencia \\
\hline $\mathbf{6}$ & La Becerrera (Niño Jesús) & $\mathbf{1 3}$ & Doña Lula \\
\hline & & $\mathbf{1 4}$ & Mixteque \\
\hline & & $\mathbf{1 5}$ & Mucumpate (Alto La Laguneta) \\
\hline
\end{tabular}

Fuente: elaborado a partir de los datos establecidos para la determinación de la muestra.

El instrumento para la recolección de datos primarios se basó en la aplicación de una entrevista estructurada dirigida a los miembros principales de los $\mathrm{CC}$ de la muestra.

\section{Diagnóstico de los CC del municipio Rangel}

Para determinar y evaluar los procedimientos y métodos utilizados en la rendición de cuentas de los CC se aplicó una entrevista estructurada que permitió levantar información sobre este proceso. Este instrumento se aplicó a los integrantes de la Unidad de Gestión Financiera, lo que permitió evaluar la metodología y el control de gestión utilizados para la rendición de cuentas de los recursos recibidos por los CC. En tal sentido, se obtuvieron los siguientes resultados:

1. Es importante verificar la formación e integración de los $\mathrm{CG}$ como un colectivo de coordinación comunitaria que especifica funciones, de los integrantes de las unidades que los componen y las responsabilidades de los mismos. En el estudio, la totalidad de los CG se encuentran constituidos e integrados adecuadamente. Lo que permite señalar que los $\mathrm{CG}$ se armaron con sus respectivas unidades de gestión y control y, para el caso de los comités de trabajo, sólo fue seleccionado un vocero para cada uno.

2. El conocimiento sobre las funciones de los CG presentes en la LCG, por parte de los miembros que forman las unidades de gestión de los mismos, es otro aspecto a considerar en el estudio. $\mathrm{Al}$ respecto la LCG establece: 
a) Administración de los recursos.

b) Promover la constitución de cooperativas.

c) Impulsar el diagnóstico y presupuesto participativo.

d) Servir de ente de inversión y crédito.

e) Realizar intermediación financiera.

f) Rendir cuenta.

Para ello, realizamos un análisis a partir de la información extraída del instrumento aplicado, en la que se señala la frecuencia de respuesta de cada una de las funciones por parte de los CG (Cuadro 5).

Cuadro 5. Cumplimiento de funciones presentes en la LCG del municipio Rangel por parroquia 2006-2008

\begin{tabular}{|c|c|c|c|c|c|c|c|}
\hline \multirow{2}{*}{ Parroquia } & \multirow{2}{*}{ Muestra } & \multicolumn{6}{|c|}{ Número de GG que conocen las funciones en la LGG } \\
\hline & & $\mathrm{al} /$ & b2/ & c3/ & $\mathrm{d} 4 /$ & e5/ & $\mathrm{f} 6 /$ \\
\hline Cacute & 6 & 5 & 2 & 5 & 2 & 2 & 5 \\
\hline Mucurubá & 8 & 7 & 3 & 7 & 2 & 2 & 7 \\
\hline Rangel & 16 & 6 & 8 & 5 & 6 & 5 & 5 \\
\hline La toma & 7 & 6 & 2 & 6 & 2 & 2 & 6 \\
\hline San Rafael & 9 & 7 & 2 & 7 & 3 & 2 & 8 \\
\hline Total & 46 & 41 & 17 & 40 & 15 & 13 & 41 \\
\hline$\%$ con respecto al total & $89 \%$ & $37 \%$ & $87 \%$ & $33 \%$ & $28 \%$ & $89 \%$ & \\
\hline
\end{tabular}

Este análisis se realiza a partir del número de respuestas positivas en cada una de las funciones que estaban presentes en la muestra de los $\mathrm{CG}$ discriminados por parroquia:

En la muestra de los CG estudiados, entre 87 y 89 \% conocían las funciones de: administración de los recursos; impulsar el diagnóstico y presupuesto participativo y rendir cuentas. Igualmente, la entrevista señala que, entre 28 y $37 \%$ de los CG de la muestra seleccionada, conocían las funciones de: promover la constitución de Cooperativas; servir de ente de inversión y crédito y realizar intermediación financiera (Cuadro 5).

Para verificar el conocimiento que tenían los miembros de la Unidad de Gestión Financiera, con respecto a las funciones de los CG, se obtuvieron los porcentajes totales de respuestas de cada una de las funciones. Posteriormente, para establecer 


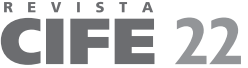

ISSN: 0124-3551 / Año 15, No 22 / enero-junio / pp. 161-187

porcentualmente las funciones desconocidas, se estableció una diferencia entre el 100 \% y los porcentajes resultantes de las funciones conocidas (Guadro 6).

Cuadro 6. Conocimiento de las funciones de los CC del municipio Rangel 2006-2008

\begin{tabular}{|l|c|c|}
\hline \multicolumn{1}{|c|}{ Funciones } & Conocidas & Desconocidas \\
\hline Administración de los Recursos & $89 \%$ & $11 \%$ \\
\hline Promover la constitución de Cooperativas & $37 \%$ & $63 \%$ \\
\hline Impulsar el diagnóstico y presupuesto participativo & $87 \%$ & $13 \%$ \\
\hline Servir de ente de inversión y crédito & $33 \%$ & $67 \%$ \\
\hline Realizar intermediación financiera & $28 \%$ & $72 \%$ \\
\hline Rendir cuenta & $89 \%$ & $11 \%$ \\
\hline Promedio para el municipio & $60,50 \%$ & $39,50 \%$ \\
\hline
\end{tabular}

Fuente: elaborado a partir de entrevista aplicada

Gráfico 1. Porcentaje total de conocimiento de funciones de los CG del municipio Rangel 2006-2008

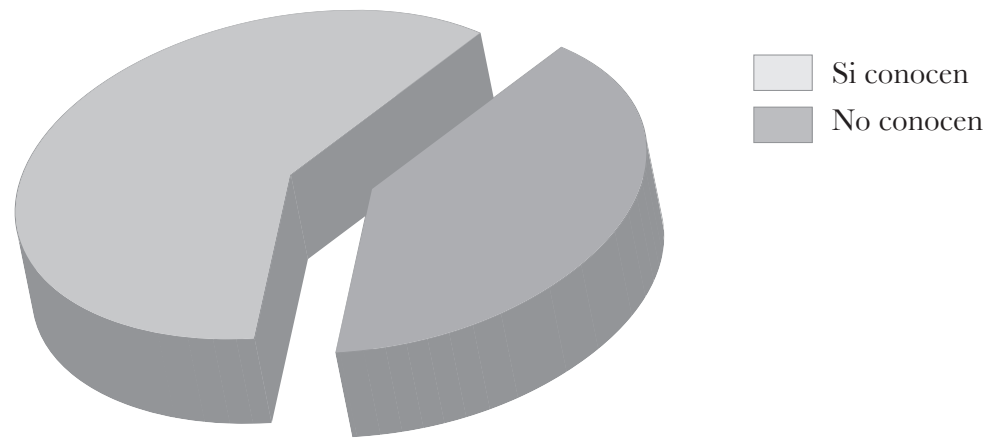

Fuente: elaborado a partir de entrevista aplicada

De los resultados se desprende que, $60 \%$ aproximadamente, de los miembros integrantes de la Unidad de Gestión Financiera conocen las funciones de los CG que están presentes en la LCG, esto significa que existe un alto nivel de desconocimiento de las mismas (Gráfico 1).

Otro de los aspectos importantes del presente trabajo es la responsabilidad del seguimiento contable. El seguimiento contable está definido como un proceso para cumplir con la función de Rendir Cuentas, lo que nos lleva a conocer las capacidades, actitudes, expectativas y satisfacciones de los representantes de la Unidad de Gestión Financiera de los CG del municipio Rangel. En este sentido, 91,30\% de los CG lleva registros 
contables, sin embargo, implica hacer un análisis de las posibilidades encontradas en el municipio, con relación a la disponibilidad de un miembro(s) u otro agente que se encargue de realizar esta función, ya que no todos los CC, tienen dentro de sus integrantes una persona que reúna el perfil adecuado para desempeñar esta función. A partir de la información obtenida se extraen las siguientes consideraciones:

El 100 \% de los CC conocían que la responsabilidad de los Registros Contables debía ser una persona que no forma parte de la Unidad de Control Social, debido a que esta función no le corresponde a esa unidad (Cuadro 7).

Cuadro 7. Encargados de realizar los Registros Contables del CG del municipio Rangel según Parroquia, 2006-2008

\begin{tabular}{|c|c|c|c|c|c|c|c|}
\hline \multirow[b]{2}{*}{ Parroquia } & \multicolumn{7}{|c|}{ ¿Quién realiza los Registros Contables en su CC? } \\
\hline & 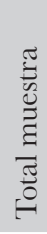 & 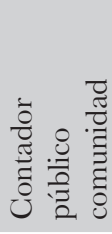 & 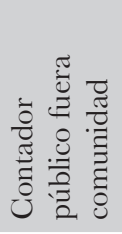 & 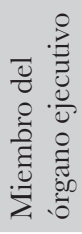 & 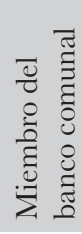 & 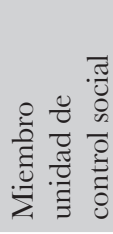 & 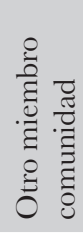 \\
\hline Cacute & 6 & 2 & 1 & 1 & 1 & 0 & 1 \\
\hline Mucurubá & 8 & 3 & 2 & 0 & 1 & 0 & 1 \\
\hline Rangel & 16 & 6 & 5 & 3 & 0 & 0 & 1 \\
\hline La Toma & 7 & 2 & 1 & 0 & 1 & 0 & 1 \\
\hline San Rafael & 9 & 3 & 2 & 0 & 2 & 0 & 2 \\
\hline Totales & 46 & 16 & 11 & 4 & 5 & 0 & 6 \\
\hline Porcentaje $(\%)$ & & $35 \%$ & $24 \%$ & $9 \%$ & $11 \%$ & $0 \%$ & $13 \%$ \\
\hline
\end{tabular}

Fuente: elaborado a partir de entrevista aplicada

Igualmente, se puede observar que $20 \%$ de las organizaciones (la suma de los miembros del órgano ejecutivo y el banco comunal), consideraban que esta responsabilidad estaba en manos de la Unidad de Gestión Financiera y el Banco Comunal. Sólo 35 \% de los $\mathrm{CG}$ entrevistados, respondieron que esta actividad era realizada por un profesional contable, que formaba parte de la comunidad y $24 \%$ de los mismos, contrataron externamente de otra comunidad, este profesional (Cuadro 7).

De acuerdo con la muestra entrevistada, $59 \%$ aproximadamente (la suma de contadores dentro y fuera de la comunidad), tenían conocimiento que la responsabilidad de los Registros Contables correspondía a un profesional contable. 
Cabe destacar que, aunque esta función recae sobre el Órgano Económico Financiero del CG, la responsabilidad de este tipo de trabajo debe estar en manos de un profesional contable, que posea las técnicas y conocimientos teóricos en el área. Por ello, 35 \% de los casos entrevistados incorporaron personal de esta profesión.

Así mismo, la totalidad de los integrantes de los CG, conocían los libros contables que debe llevar el Banco Comunal. En tal sentido, 91 \% de los entrevistados conocían las exigencias y requerimientos de los soportes y/o comprobantes de los egresos del CC para la rendición de cuentas.

En la totalidad de los casos entrevistados, los Órganos Económicos Financieros de los CG estaban conformados como una Cooperativa Banco Comunal. De acuerdo con la LCC, para obtener los beneficios de la misma, las Cooperativas deben presentar semestralmente los Estados Financieros de la Cooperativa.

Los CG que cumplen con este requisito, obtienen una Certificación de Fiel Cumplimiento por parte de la Superintendencia Nacional de Cooperativas (SUNACOOP).

Cuadro 8. Certificación de Fiel Cumplimiento (SUNACOOP) en los CG del municipio Rangel por parroquia 2006-2008

\begin{tabular}{|l|c|c|c|c|}
\hline \multicolumn{1}{|c|}{ Parroquia } & \multicolumn{4}{|c|}{ SUNACOOP expidió el certificado de Fiel Cumplimiento } \\
\hline & Total muestra & Respuesta positiva & Respuesta negativa & $\begin{array}{c}\text { Desconoce el } \\
\text { procedimiento }\end{array}$ \\
\hline Cacute & 6 & 3 & 2 & 1 \\
\hline Mucurubá & 8 & 4 & 3 & 1 \\
\hline Rangel & 16 & 8 & 6 & 2 \\
\hline La Toma & 7 & 4 & 2 & 1 \\
\hline San Rafael & 9 & 5 & 3 & 1 \\
\hline Total municipio & 46 & 24 & 16 & 6 \\
\hline Porcentaje & & $52 \%$ & $35 \%$ & $13 \%$ \\
\hline
\end{tabular}

Fuente: elaborado a partir de entrevista aplicada.

Con base a los requerimientos de SUNACOOP, 13 \% de los CG desconocían el procedimiento a seguir para el cumplimiento de los requisitos exigidos por la misma. Así mismo, 35 \% no habían tramitado la solicitud de Fiel Cumplimiento por dos razones: aún estaban ejecutando recursos o tenían algún problema para realizar este procedimiento, que no definieron (Cuadro 8).

Así mismo, 52 \% de los entrevistados habían realizado los trámites ante SUNACOOP, para la obtención del Certificado de Fiel Cumplimiento (Gráfico 2). 
Gráfico 2. Certificación de Fiel Cumplimiento (SUNACOOP) en los CC del municipio Rangel 2006-2008

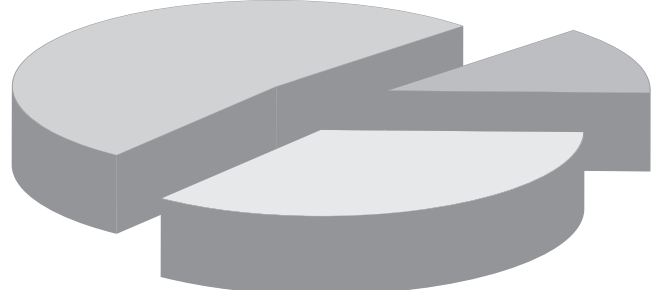

No Cumplió el procedimiento

Si cumplió el procedimiento

Desconoce el procedimiento

Fuente: elaborado a partir de entrevista aplicada

Es importante destacar que, el cumplimiento de este requisito es el aval que sustenta el estado financiero del CG, y en ellos se refleja el uso de los recursos otorgados para el financiamiento de las obras del mismo. Por esta razón, este trámite se convierte en una de las garantías que tiene el CG para que siga funcionando, pues sin este no pueden realizar nuevas solicitudes de financiamiento a organismos del Estado venezolano.

La Capacidad de Gestión de los recursos otorgados a los CC se evaluó a través de las actividades de seguimiento que estos realizan (Cuadro 9).

Cuadro 9. Actividades de Seguimiento que realizan los CC del municipio Rangel por Parroquia 2006-2008

\begin{tabular}{|c|c|c|c|c|c|c|}
\hline \multirow[t]{2}{*}{ Parroquia } & \multicolumn{6}{|c|}{ Actividades de seguimiento que realizan los CC } \\
\hline & $\begin{array}{c}\text { Total } \\
\text { muestra }\end{array}$ & $\begin{array}{c}\text { Acta de } \\
\text { inicio }\end{array}$ & $\begin{array}{c}\text { Avance } \\
\text { de } \\
\text { ejecución }\end{array}$ & $\begin{array}{l}\text { Registro } \\
\text { fotográfico de } \\
\text { cada fase }\end{array}$ & $\begin{array}{l}\text { Informe de } \\
\text { resultados } \\
\text { inspeccion }\end{array}$ & $\begin{array}{c}\text { Informe } \\
\text { seguimiento y } \\
\text { avance de la obra }\end{array}$ \\
\hline Cacute & 6 & 6 & 1 & 1 & 2 & 0 \\
\hline Mucurubá & 8 & 5 & 2 & 4 & 1 & 1 \\
\hline Rangel & 16 & 14 & 12 & 9 & 9 & 7 \\
\hline La toma & 7 & 7 & 1 & 2 & 1 & 2 \\
\hline San Rafael & 9 & 7 & 1 & 2 & 2 & 0 \\
\hline Total municipio & 46 & 39 & 17 & 18 & 15 & 10 \\
\hline Porcentaje & & $85 \%$ & $37 \%$ & $39 \%$ & $33 \%$ & $22 \%$ \\
\hline
\end{tabular}

Fuente: elaborado a partir de entrevista aplicada 


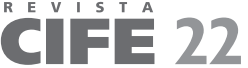

ISSN: 0124-3551 / Año 15, No 22 / enero-junio / pp. 161-187

De la evaluación realizada se pudo verificar que 85 \% de los CC realizaban un Acta de Inicio, pero solo $37 \%$ de los mismos, hacían seguimientos de avances de ejecución de la obra y, $22 \%$ realizaba el informe escrito del avance (Cuadro 9 y Gráfico 6).

Gráfico 6. Actividades de seguimiento que realizan los CC del municipio Rangel por parroquia 2006-2008

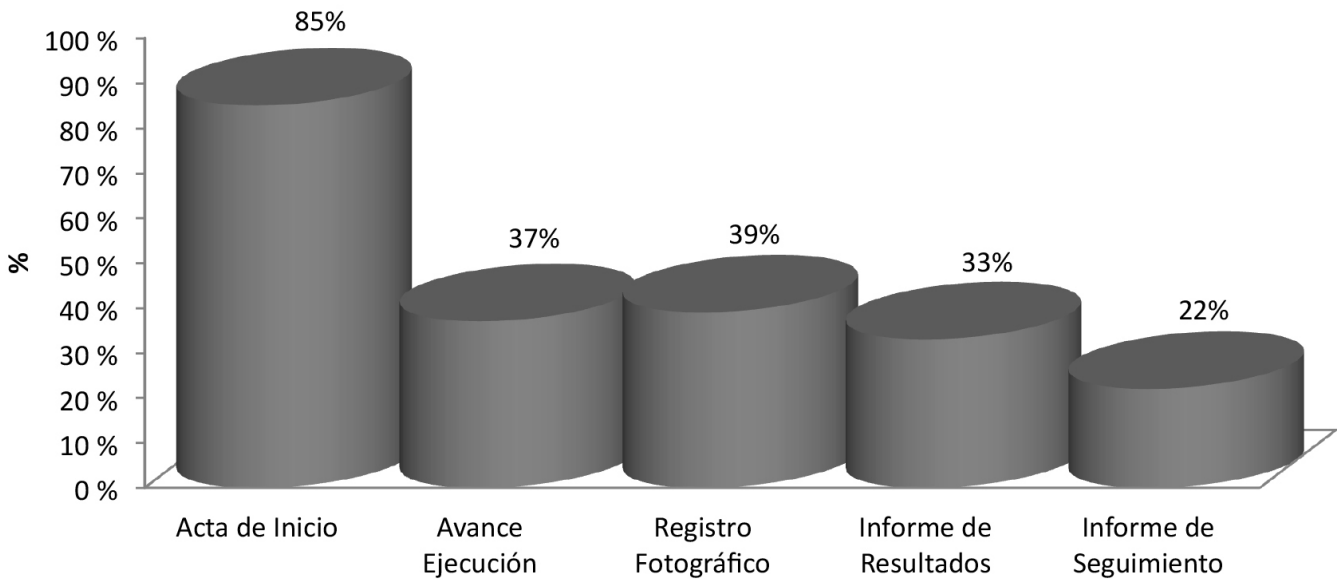

Fuente: elaborado a partir de entrevista aplicada

En cuanto al registro fotográfico del avance de la obra, se encontró que sólo 39 \% de los CC, conservan los registros fotográficos de las obras (Cuadro 9 y Gráfico 6). Así mismo, sólo $33 \%$ de los CC conservan los informes de resultado final de la obra, (Cuadro 9 y Gráfico 6).

Otras de las actividades de seguimiento de los CG fueron revisadas con las respuestas de la aplicación del instrumento, de ellos se obtuvo que las actividades como: control de partidas presupuestarias y cronograma de desembolsos, son poco conocidas por los integrantes de la Unidad de Gestión Financiera de los GG.

Así mismo, el uso de los recursos financieros, los que se realizan efectuando el desembolso cuando es requerido y sin seguir un cronograma de desembolsos, son controlados a través de una cuenta corriente a nombre del Banco Comunal, la cual realiza sus conciliaciones bancarias (Cuadro 10). 
Cuadro 11. Actividades de seguimiento en el uso de los recursos de los CC por parroquia 2006-2008

\begin{tabular}{|c|c|c|c|c|c|c|}
\hline \multirow[t]{2}{*}{ Parroquia } & \multicolumn{6}{|c|}{ La comunidad verifica el uso de recursos atendiendo a: } \\
\hline & 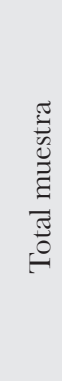 & 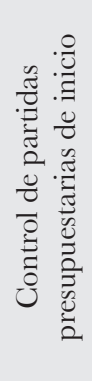 & 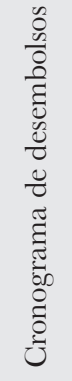 & 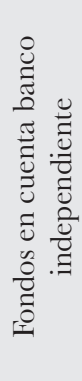 & 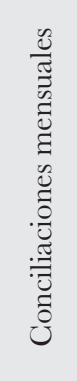 & 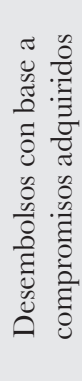 \\
\hline Cacute & 6 & 0 & 0 & 6 & 6 & 0 \\
\hline Mucurubá & 8 & 0 & 0 & 8 & 8 & 0 \\
\hline Rangel & 16 & 1 & 4 & 14 & 13 & 0 \\
\hline La toma & 7 & 1 & 1 & 7 & 7 & 0 \\
\hline San Rafael & 9 & 0 & 1 & 8 & 9 & 0 \\
\hline Total municipio & 46 & 2 & 6 & 43 & 43 & 0 \\
\hline Porcentaje & & $4 \%$ & $13 \%$ & $93 \%$ & $93 \%$ & $0 \%$ \\
\hline
\end{tabular}

Fuente: elaborado a partir de entrevista aplicada

\section{Análisis estratégico de los CC del municipio Rangel}

A partir de los resultados de la encuesta aplicada se realizó un diagnóstico estratégico de los CG del municipio Rangel (2006-2008). Para ello, se elaboró un análisis FODA (Fortalezas, Oportunidades, Debilidades y Amenazas) con la finalidad de diseñar un conjunto de estrategias que permitan a estas organizaciones realizar seguimiento eficiente a su desempeño financiero y así un mejor proceso de rendición de cuentas (Esquema 2). 
Esquema 2. Análisis FODA de los CG del municipio Rangel 2006-2008

\section{Fortalezas}

Cohesión de las organizaciones del municipio que facilita e incrementa la participación comunitaria.

Sentido de pertenencia de los miembros de los CG con estas organizaciones.

Voluntad por parte de los integrantes de la comunidad para realizar un buen desempeño.

Capacidad de gestión para emprender proyectos.

\section{Oportunidades}

Interés del Gobierno nacional y subnacional para el fomento de estas organizaciones.

Disponibilidad de recursos financieros por parte del Estado para la ejecución de proyectos de los CC.

Apoyo de las organizaciones del Estado para el funcionamiento de los CC (Alcaldía, INDER1, INIA2, entre otros.)

\section{Debilidades}

Falta de seguimiento y control a los CC por parte de los entes encargados de estas funciones.

Desconocimiento de funciones por parte de los dirigentes de los CC.

Falta de evaluación de gestión de resultados por parte de los miembros de los $\mathrm{CC}$.

Expectativas insatisfechas por parte de la comunidad a partir de los resultados esperados de los CC.

$1 /$ Instituto Nacional de Desarrollo Rural (INDER)

2/ Instituto Nacional de Investigación y Tecnología Agraria y Alimentaria (INIA)

Fuente: elaboración propia a partir de entrevista aplicada

Este análisis permitió definir en estas estructuras organizativas las potencialidades, los desafíos, los riesgos a los que están sometidos y las principales advertencias.

Una vez realizado el análisis FODA y teniendo en cuenta todos los aspectos mencionados en el esquema 2, observamos: 


\section{Potencialidades}

Las potencialidades del municipio tienen que ver con la cohesión en las organizaciones y el sentido de pertenencia encontrado en los $\mathrm{CC}$, lo que ha permitido un incremento de la participación comunitaria para acceder a recursos del estado.

\section{Advertencias}

Las advertencias más significativas encontradas tienen que ver con el desempeño de los CG dentro del municipio:

1. La ausencia de acompañamiento, los bajos niveles interrelación y la falta de controles entre los CG y las unidades controladoras del estado, pueden ocasionar el fracaso de los mismos. Por esta razón, los CG deben contar con una organización que permita el acompañamiento de la gestión financiera, durante la ejecución de los proyectos.

2. La falta de evaluación de gestión de resultados de los $\mathrm{CC}$, la percepción de la comunidad de insatisfacción, con relación al desempeño de estas organizaciones, y la escasa capacitación suministrada en el área de rendición de cuentas hacen vulnerables estas organizaciones en su funcionamiento y permanencia.

\section{Riesgos:}

Los riesgos más importantes a los que están expuestos los CG son:

1. A pesar que, existe cohesión entre este tipo de organizaciones, los vacíos legales en relación con la figura jurídica de la conformación de los $\mathrm{C}$ C pone en riesgo el manejo administrativo y en el tiempo la permanencia de los mismos.

2. En el municipio existe una voluntad por parte de los integrantes de los CG para realizar un buen desempeño, pero la falta de capacitación en el área de rendición de cuentas y el acompañamiento de una organización dedicada exclusivamente a los mismos pone en riesgo la permanencia de éstos en el tiempo.

\section{Desafíos}

El principal desafío que enfrentan los CG es compensar la falta de evaluación de gestión de resultados.

Para contrarrestar las debilidades, amenazas y aprovechar las fortalezas y las oportunidades de los CG se deben crear estrategias. Es importante resaltar que el éxito de las estrategias requiere del apoyo institucional a través del acompañamiento por parte del Estado a los CG. Para ello: 
Estrategias de Acompañamiento: se debe realizar material divulgativo de distribución a estas organizaciones que contenga lineamientos para realizar una adecuada rendición de cuentas, en donde se incluyen todas las etapas de la misma. Para ello, el Estado debe realizar la difusión de estos folletos a todas las organizaciones creadas en las respectivas jurisdicciones.

Estrategias de Gapacitación: realizar talleres de capacitación relacionados con la rendición de cuentas.

Estrategias de implantación: realizar evaluación de la ejecución a través del acompañamiento y la capacitación (gestión de resultados).

\section{Conclusiones}

La conformación y funcionamiento de este tipo de organizaciones está sujeta a factores técnicos y a elementos de capital social importantes para el desempeño y permanencia de las mismas. En tal sentido, es este estudio la conformación de los CG del municipio Rangel se encuentra estructurada con miembros que fortalecen un conjunto de valores relacionados con: honestidad, responsabilidad compartida, integridad y solidaridad. En cualquier organización estos valores permiten establecer un alto nivel de compromiso, lo que contribuye con el cumplimiento de sus funciones. Sin embargo, la rendición de cuentas es una función que no se realiza de acuerdo con lo dispuesto por la normativa de la administración pública y la LCC, debido a vacios legales para su cumplimiento.

Así mismo, existe un alto sentido de pertenencia en la conformación y gestión de los GC, estas organizaciones se derivan de una vocación territorial asociada a la actividad agrícola, la cual tiene raíces ancestrales. Esta condición, ha permitido que se consolide un capital social que fortalece a estas organizaciones y al municipio. A partir de esta fortaleza se pueden atender y solucionar los problemas de la comunidad. Así mismo, este perfil de organización permite a los $\mathrm{CG}$ acceder al financiamiento para realizar sus proyectos.

Las formas de participación de los miembros de los CG ha variado en distintos roles. Entre ellos destaca la participación como mano de obra en diferentes proyectos, lo que ha permitido culminar los mismos y ahorrar recursos financieros. Sin embargo, esta práctica no se ha incorporado de manera permanente debido a que la mayoría de los integrantes de estos CG, son jornaleros y trabajadores del campo, por lo que se les dificultaba disponer de un día de su trabajo, para realizar estas actividades.

Igualmente, en las organizaciones analizadas, se encontró que, el financiamiento de las obras era asumido casi en su totalidad por el Estado venezolano, solo se encontró una muy pequeña porción de financiamiento otorgada por los aportes de la comunidad. Esta forma de autofinanciamiento consta de una pequeña porción de dinero que aporta cada integrante del CG para contribuir con los gastos administrativos. 
El desconocimiento de algunas de las funciones que se encuentran en la LCG, ponen en desventaja a estas organizaciones para fomentar actividades socio productivas dentro del municipio. Los CG surgen como una nueva tipología de organización a partir de la participación comunitaria, lo que obligó a los miembros de los mismos a tomar decisiones que no han proporcionado un eficiente desempeño en materia de rendición de cuentas, debido al desconocimiento de procesos administrativos. Esta falta de conocimiento sobre el manejo de estas nuevas organizaciones, ocasionó demoras en los procesos de rendición de cuentas, requisito indispensable para acceder a nuevos financiamientos.

Algunos de los factores que ponen en riesgo a los $\mathrm{CC}$ en el tiempo son los vacíos legales en relación con la figura jurídica de la conformación y la incapacidad para desarrollar los procesos que permitan cumplir con la rendición de cuentas por parte de los mismos. Una de las evidencias encontradas al respecto, fue la demora al obtener el Certificado de Fiel Cumplimiento de SUNACOOP, documento necesario para acceder a futuros financiamientos.

Es importante destacar que, las responsabilidades e implicaciones legales que en ella se encontraban, obligaron a los representantes de estas organizaciones a establecer sinergias, con los organismos del Estado y sus funcionarios, para trabajar en forma conjunta.

Los CG entregan una relación de cuentas de los recursos financieros utilizados, en donde obvian aspectos importantes de la rendición de cuentas como son: seguir el cronograma establecido, la evaluación entre los interesados y afectados en el proyecto durante su ejecución, la revisión de los aspectos positivos y negativos para buscar oportunidades de mejora y el seguimiento a las actividades planificadas.

A partir de lo anteriormente expuesto se desprende que, el mal manejo en la capacidad de gestión pueden generar insatisfacción por parte los integrantes de la comunidad de acuerdo con los resultados de las obras ejecutadas.

$\mathrm{Al}$ realizar una adecuada rendición de cuentas, se podrían evitar los conflictos entre los grupos de interés y los costos elevados en las obras. El manejo inadecuado de la misma puede afectar el desempeño de estas organizaciones, debilitar su capacidad de gestión y la permanencia de las mismas en el tiempo. Por ello, el Estado debe asumir un rol más formal y activo en el seguimiento de estas organizaciones. En tal sentido, este apoyo institucional puede fortalecer y potenciar el capital social que posee la localidad y las organizaciones comunitarias. 


\section{Referencias}

Acevedo, D. (2007). De la medición a la rendición de cuentas: Un enfoque estratégico. Caracas: Alpha Omega Estudio Creativo C. A.

Constitución de La República Bolivariana de Venezuela. (1999). Gaceta Oficial de la República Bolivariana de Venezuela 36860 (diciembre 30, 1999).

Contraloría General de La República Bolivariana de Venezuela (2004). "El control ciudadano: Experiencias de las entidades fiscalizadoras superiores." Revista de Control Fiscal, 156, pp. 11-46.

Decreto 287-27 de Junio de 1958. Disponible: http://www.ocepre.gov.ve/que\%20es\%20 onapre/dec287.html. Consulta: 2008, enero, 21.

Decreto 49230 de diciembre de 1958. Disponible: http://www.ocepre.gov.ve/que\%20 es\%20onapre/dec492.html. Consulta: 2008, enero, 21.

Delgado, A. (1995). “Contribución del análisis de políticas a la gestión pública.” Cuestiones Politicas, 15, pp. 6-11.

Herrera, H. (2008). Manual de organización de los consejos comunales. Caracas: Editorial Panapo de Venezuela.

Hurtado, J. (2000). Metodología de la investigación holística. Caracas: Fundación SYPAL.

Ley Especial de Asociaciones Cooperativas (2001). Gaceta Oficial de la República Bolivariana de Venezuela 37.285 (septiembre 18, 2001).

Ley Orgánica de la Contraloría General de la Republica y el Sistema Nacional del Control Fiscal (2001). Gaceta Oficial de la República Bolivariana de Venezuela 37347 (diciembre 17, 2001).

Ley Orgánica del Poder Publico Municipal (2005). Gaceta Oficial de la República Bolivariana de Venezuela 38.204 (junio 8, 2005).

Ley de los Consejos Locales de Planificación Pública (2002). Gaceta Oficial de la República Bolivariana de Venezuela 37463 junio 12, 2002).

Ley Especial de Consejos Comunales (2006). Gaceta Oficial Extraordinaria de la República Bolivariana de Venezuela 5806 (abril 10, 2006).

Ley Especial de Asociaciones Cooperativas (2001). Gaceta Oficial de la República Bolivariana de Venezuela 37.285 (septiembre 18, 2001). 
Moreno, M. (2007). "La descentralización en el banquillo o ausencia del 'principal'." [Documento en línea] Disponible: http://firmasfaces.blogia.com/2007/ 041303-ladescentralización-en-el banquillo-o-ausencia del principal-maria-antonia-more.php. Consulta: 2008, enero, 19.

Paredes F. (2006). El presupuesto público: Aspectos teórico-prácticos. Mérida (Venezuela): Publicaciones del Vicerrectorado Académico.

Romero, R. (2007). Los consejos comunales más allá de la utopía. Maracaibo: Universidad del Zulia.

Sánchez, J. (2002). "Pasado, presente y futuro de la descentralización en Venezuela." Provincia, 9, pp. 20-33.

Tamayo, M. (1988). El proceso de la investigación científica. (2ª ${ }^{2}$ Ed.) México, D. F.: Editorial Limusa, S.A.

Universidad Pedagógica Experimental Libertador (2003) Manual de trabajos de grado de especialización, maestría y tesis doctorales. Tercera edición. FEDUPEL. Venezuela. 
